\title{
Analysis of Theoretical Models for Nonlinear Fitting of Experimental Data: Dielectric Permittivity of $\mathrm{Pb}_{4.95} \mathrm{Ge}_{3} \mathrm{O}_{11}$ Crystals
}

\author{
Yu. G. Klymovych \\ Optoelectronics and Information Technologies Department \\ Ivan Franko National University of Lviv \\ Lviv, Ukraine \\ yuriy.klymovych@gmail.com
}

\author{
A. I. Kashuba \\ Optoelectronics and Information Technologies Department \\ Ivan Franko National University of Lviv \\ Lviv, Ukraine \\ andriykashuba07@gmail.com
}

\author{
O. S. Kushnir \\ Optoelectronics and Information Technologies Department \\ Ivan Franko National University of Lviv \\ Lviv, Ukraine \\ o.s.kushnir@lnu.edu.ua
}

\begin{abstract}
We implement nonlinear fitting and select the best theoretical model for description of experimental data, basing on the programming language Java. This is achieved using the Levenberg-Marquardt algorithm and the bootstrapping for determining confidence intervals for the fitting parameters. The main technical procedures are illustrated on the temperature dependence of dielectric permittivity of non-stoichiometric LG crystals in their paraelectric phase. Our results confirm that the model incorporating a temperature-independent dielectric background and a normal spatial distribution of Curie points assumed for the case of diffused phase transitions can be used as a statistically significant hypothesis.
\end{abstract}

Index Terms-Nonlinear fitting; mathematical models; residuals; bootstrapping; phase transitions; dielectric permittivity; lead germanate; non-stoichiometry

\section{INTRODUCTION AND THEORETICAL MODELS}

The temperature behavior of physical characteristics near the points of ferroelectric phase transitions attracts considerable attention of researchers in the field of materials science and physics of semiconductors and dielectrics. It is known that the temperature dependence of the dielectric permittivity of crystalline ferroics can be influenced by many factors, one way or another associated with the phase transition: thermal fluctuations, long-range dipole interactions, heterogeneity of a crystal, structural disordering due to defects, diffused nature of the phase transition, contributions of domain walls and some other factors [1-5]. Then finding out the best theoretical model that describes dominant contributions to the temperature dependence of dielectric permittivity turns out to be a difficult problem. Due to many influencing effects, a standard linear fitting is no good here. It must be replaced by much more cumbersome techniques of nonlinear fitting (see, e.g., $[6,7]$ ), of which performance and efficiency represent a separate subject.

In this work we seek to find the optimal theoretical model for the temperature behavior of dielectric permittivity on the example of non-stoichiometric lead germanate (LG) crystals, $\mathrm{Pb}_{4.95} \mathrm{Ge}_{3} \mathrm{O}_{11}$. These crystals have been grown at the Oles Honchar Dnipro National University (Ukraine). Here the nonstoichiometry is concerned with the lead atoms and is given by the parameter $\mathrm{x}=4.95$, instead of the stoichiometric value $\mathrm{x}=5$. The above structural modification has been introduced in order to control the ionic and hole conductivity components and to improve the photorefractive properties of LG [8]. The experimental data have been measured for the polar axis $z$ at the working frequency $1 \mathrm{kHz}$ (see [9]). The temperature dependence of the dielectric permittivity is presented in Fig. 1. Below we focus on the quantitative interpretation of this data.

The following theoretical models have been tested to describe quantitatively the dielectric permittivity in the paraelectric phase:

$$
\begin{aligned}
& \varepsilon(T)=C /\left|T-T_{C}\right|, \\
& \varepsilon(T)=C /\left|T-T_{C}\right|^{\gamma}, \\
& \varepsilon(T)=C\left|T-T_{C}\right|^{-1}\left[\ln \left(a+t_{0} /\left|T-T_{C}\right|\right)\right]^{1 / 3}, \\
& \varepsilon(T)=\varepsilon_{b}+\varepsilon_{\max }\left[1+\left|T-T_{C}\right|^{\gamma} /\left(2 \delta^{2}\right)\right]^{-1} .
\end{aligned}
$$


Here $T_{C}$ is the phase transition temperature, and the constants $C, a, t_{0}, \varepsilon_{b}, \varepsilon_{\max }, \gamma$ and $\delta$ represent fitting parameters (see [1-5]). Note that some conclusions concerning the optimal mathematical models for the dielectric permittivity of LG can be drawn even with no fitting. For instance, Fig. 2 evidences that the simplest model (1) is insufficient. However, discriminating among the models (2)-(4) is still not trivial.

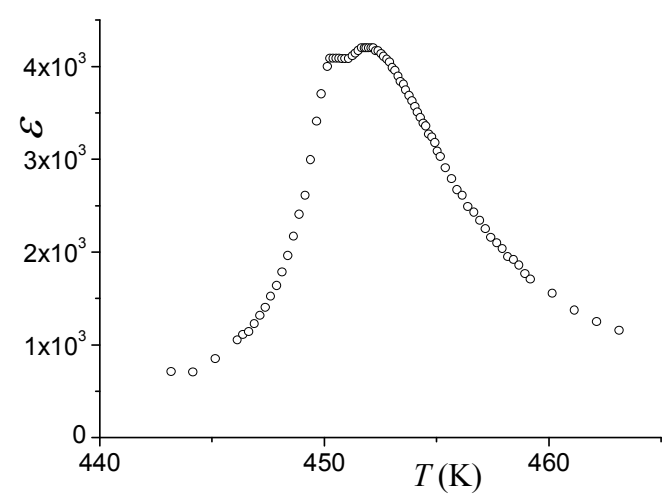

Figure 1. Temperature dependence of dielectric permittivity $\varepsilon$ for nonstoichiometric LG

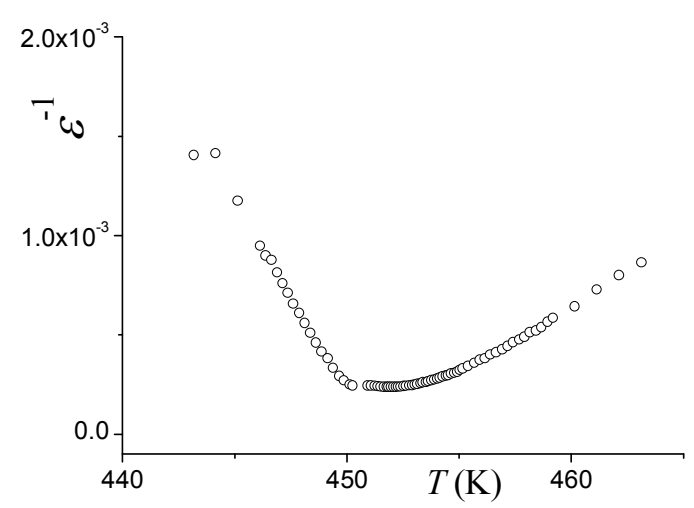

Figure 2. Temperature dependence of inverse dielectric permittivity $\varepsilon^{-1}$ for non-stoichiometric LG

\section{METHODS OF ANALYSIS}

For the sake of conciseness, below we present only the main results of our numeric analysis of the data shown in Fig. 1. It has turned out that the best theoretical model is that given by formula (4). It takes into account the background $\varepsilon_{b}$ and assumes a normal spatial distribution of the Curie points inside a crystal, which corresponds to a specific case of diffused phase transitions. The corresponding arguments are based upon the goodness of fit and a number of appropriate characteristics. As a consequence, we will omit any comparison of all the models (1)-(4) and concentrate only at the data obtained with the model (4).
The main technical problem has been the nonlinear fitting and the accompanying accuracy and reliability estimations. We have resolved this using a Levenberg-Marquardt algorithm. The latter has been implemented using the programming language Java.

To determine the error margins for the fitting parameters, we have employed a bootstrapping technique. In particular, a method of random sampling of the fitting residuals has been used for bootstrapping. It works as follows. First, the fitting of the experimental data $\left(x_{i}, y_{i}\right)$ (with $i=1, \ldots, n$ being the number of experimental data points) is carried out in accordance with the chosen theoretical model, and the fitted ordinate values $\hat{y}_{i}$ and the residuals $\hat{\xi}_{i}=y_{i}-\hat{y}_{i}$ are obtained. Then for each of the pairs $\left(x_{i}, y_{i}\right)$ we randomly add the fitting residual $\hat{\xi}_{j}$. In other words, we create a new synthetic dataset $y_{i}^{*}=y_{i}+\hat{\xi}_{j}$, where $j$ for each $i$ is chosen randomly from the list $i=1, \ldots, n$. After that, we repeat the fitting procedures for the synthetic data set $\left(x_{i}, y_{i}^{*}\right)$. In this study, we have obtained 2000 synthetic data sets and used them to determine the errors of the fitting parameters.

\section{RESULTS AND DISCUSSION}

The results of the nonlinear fitting concerned with the model (4) and applied to the case of paraelectric phase of LG are shown in Fig. 3. Here the fit has been performed in a slightly wider temperature region around the phase transition point than that satisfying the condition $\varepsilon>(2 / 3) \varepsilon_{\max }$ [5]. Namely, this has been done for the points in the paraelectric phase which satisfy a less strong condition, $\varepsilon>0.3 \varepsilon_{\max }$.

Fig. 4 shows the temperature behavior of so-called fitting residuals, i.e. differences between the experimental $\left(\varepsilon_{\exp }\right)$ and theoretical $\left(\varepsilon_{\mathrm{th}}\right)$ values of the dielectric constant for all the temperature points under analysis.

Table I summarizes the initial parameters that characterize the fitting, with no bootstrapping performed. These are the parameters entering formula (4) and the mean-square deviation (SD) between the theory and the experiment.

TABLE I. INITIAL PARAMETERS OF NONLINEAR FITTING WITH FORMULA (4) OF THE TEMPERATURE DEPENDENCE OF DIELECTRIC PERMITTIVITY FOR THE PARAELECTRIC PHASE OF NON-STOICHIOMETRIC LG (BEFORE BOOTSTRAPPING): THE VALUES OF THE FITTING PARAMETERS AND THE MEANSQUARE THEORY-EXPERIMENT DEVIATION

\begin{tabular}{|l|l|c|c|}
\hline \multirow{2}{*}{} & \multicolumn{3}{|c|}{ Model (4) } \\
\cline { 2 - 4 } & \multicolumn{1}{|c|}{ Parameter } & Notation & Value \\
\hline & Dielectric background & $\varepsilon_{b}$ & 474.69 \\
\hline & Maximum permittivity measured & $\varepsilon_{\max }$ & 3721.82 \\
\hline & $\begin{array}{l}\text { Phase transition temperature } \\
\text { Critical index of dielectric } \\
\text { susceptibility }\end{array}$ & $\gamma$ & 452.08 \\
\hline & $\begin{array}{l}\text { Diffusion coefficient of phase } \\
\text { transition }\end{array}$ & $\delta, \mathrm{K}^{\gamma / 2}$ & 2.83 \\
\hline & $\begin{array}{l}\text { Mean-square deviation } \\
\mathrm{SD}\end{array}$ & 9075 \\
\hline
\end{tabular}


TABLE II RESULTS OF THE WALD-WOLFOVITZ TEST

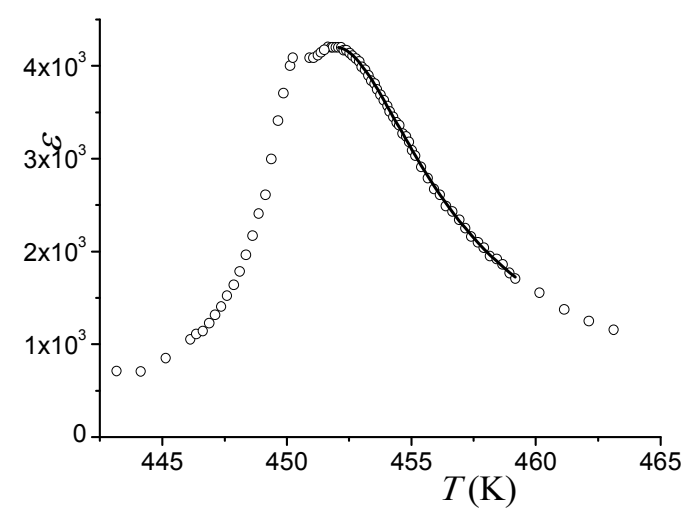

Figure 3. Nonlinear fit of the temperature dependence of dielectric permittivity $\varepsilon$ for non-stoichiometric LG in the paraelectric phase: solid line corresponds to the model (4)

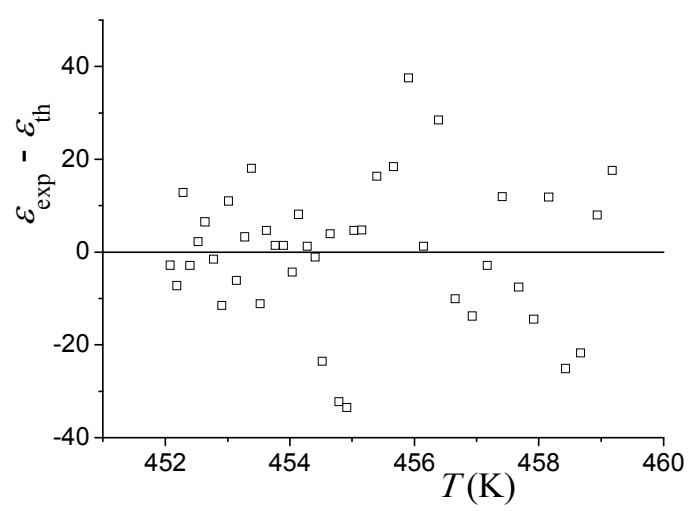

Figure 4. Temperature dependence of residuals $\left(\varepsilon_{\mathrm{exp}}-\varepsilon_{\mathrm{th}}\right)$ obtained in the nonlinear fitting of temperature dependence of the dielectric permittivity $\varepsilon$ for non-stoichiometric LG in the paraelectric phase

A Wald-Wolfowitz test has been used to study the residuals of the fitting. This test consists in comparing the expected number of batches $R=\frac{2 n_{p} n_{n}}{n_{p}+n_{n}}+1$ with the observed number of batches $n_{R}$ in a sequence of residuals, as well as determining the dispersion value $\sigma_{R}^{2}=\frac{2 n_{p} n_{n}\left(2 n_{p} n_{n}-n_{p}-n_{n}\right)}{\left(n_{p}+n_{n}\right)^{2}\left(n_{p}+n_{n}-1\right)}$, with $n_{p}$ and $n_{n}$ being the number of positive and negative residuals. Basing on these three parameters, a standard normal deviation $Z=\frac{n_{R}-(R \pm 0.5)}{\sigma_{R}}$ can be determined, which in fact represents a corresponding $\mathrm{Z}$-score. Note that the correction +0.5 must be used if the condition $n_{R}<R$ is fulfilled. In the contrary case $\left(n_{R}>R\right)$ the correction is equal to -0.5 . The results of the Wald-Wolfowitz test are presented in table II.

\begin{tabular}{|c|c|c|}
\hline \multicolumn{3}{|c|}{ Model (4) } \\
\hline Parameter & Notation & Value \\
\hline Number of positive residuals & $n_{p}$ & 23 \\
\hline Number of negative residuals & $n_{n}$ & 19 \\
\hline Observed number of batches & $n_{R}$ & 22 \\
\hline Expected number of batches & $R$ & 21.81 \\
\hline Dispersion & $\sigma_{R}$ & 3.17 \\
\hline Standard normal deviation & $Z$ & 0.22 \\
\hline Probability associated with $Z$ & $P$ & 0.587 \\
\hline
\end{tabular}

TABLE III PARAMETERS OF NONLINEAR FITTING, WITH FORMULA (4), OF THE TEMPERATURE DEPENDENCE OF DIELECTRIC PERMITTIVITY FOR THE NONSTOICHIOMETRIC LG IN THE PARAELECTRIC PHASE (AFTER BOOTSTRAPPING): THE FITTING PARAMETERS, THEIR CONFIDENCE INTERVALS, AND THE MEANSQUARE DEVIATION THEORY-EXPERIMENT

\begin{tabular}{|c|c|c|c|}
\hline \multirow{2}{*}{} & \multicolumn{3}{|c|}{ Model (4) } \\
\cline { 2 - 4 } & Parameter notation & Value & $\begin{array}{c}\text { Confidence } \\
\text { interval }\end{array}$ \\
\hline & $\varepsilon_{b}$ & 474.69 & $594.86-597.94$ \\
\hline & $\varepsilon_{\max }$ & 3721.82 & $3605.15-3607.88$ \\
\hline & $T_{C}, \mathrm{~K}$ & 452.08 & $451.98-451.98$ \\
\hline$\gamma$ & 1.76 & $1.85-1.85$ \\
\hline & $\delta, \mathrm{K}^{\gamma / 2}$ & 2.83 & $2.9791-2.984$ \\
\hline & $\mathrm{SD}$ & \multicolumn{3}{|c|}{9075} \\
\hline
\end{tabular}

The resultant fitting data is gathered in table III. Finally, the histograms that correspond to the probability density functions found for all of the fitting parameters entering formula (4) are shown in Fig. 5.
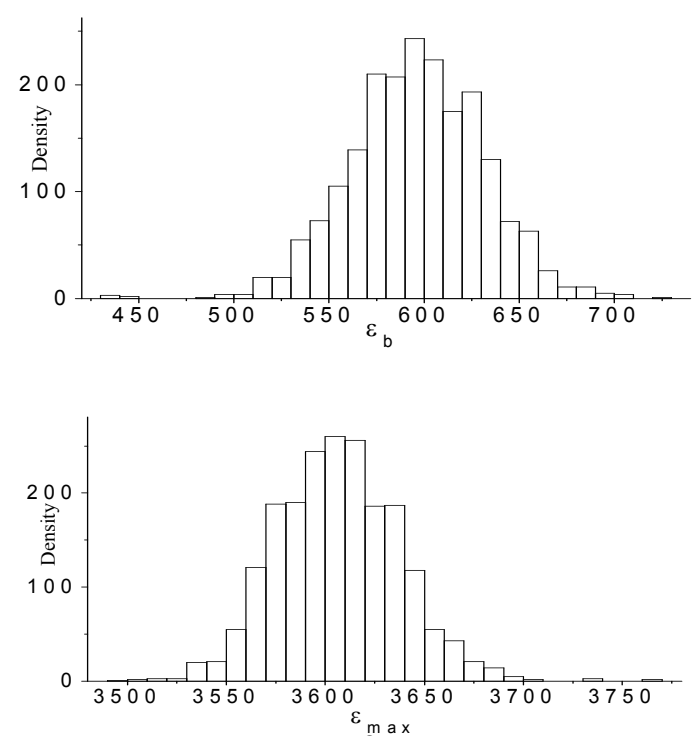

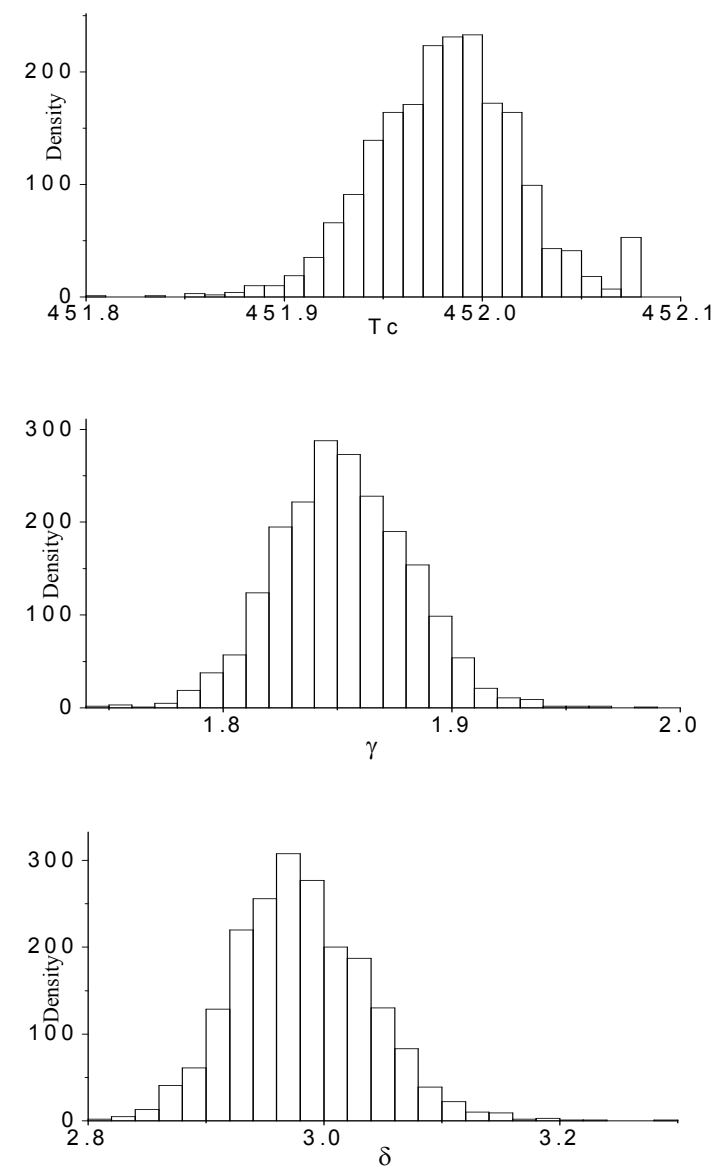

Figure 5. Histograms of probability density for the parameters of nonlinear fitting of the temperature dependence of dielectric permittivity $\varepsilon$ for nonstoichiometric LG in the paraelectric phase (the number of data sets in the statistical sample is equal to 2000)

Issuing from the results obtained above, one can state that the theoretical model (4) taking into account a temperatureindependent dielectric background and a normal spatial distribution of the Curie points occurring in the case of diffused phase transitions is statistically significant and can be used for quantitative description of the dielectric permittivity of our non-stoichiometric LG crystals.

\section{CONCLUSIONS}

In the present work we have implemented in a consistent manner all of the nonlinear fitting procedures for selecting the best mathematical model that describes the experimental data. An original program for this purpose has been written using the language Java. The technical means based on the Levenberg-Marquardt algorithm have been used. A known bootstrapping procedure has been employed for determining the confidence intervals for the fitting parameters. The procedures have been illustrated on the example of temperature dependence of the dielectric permittivity measured for the non-stoichiometric LG crystals with $\mathrm{x}=4.95$ in their paraelectric phase. The data obtained by us confirm that the theoretical model given by formula (4) can be used as a statistically significant model when describing the temperature behavior of the dielectric properties of nonstoichiometric LG crystals. This indicates that nonstoichiometric crystals can, in principle, manifest a relaxortype behavior.

\section{ACKNOWLEDGMENT}

The authors express their gratitude to Dr. Ermakov A. S. (Oles Honchar Dnipro National University, Dnipro, Ukraine), in particular for growing non-stoichiometric $\mathrm{Pb}_{4.95} \mathrm{Ge}_{3} \mathrm{O}_{11}$ crystals, supplying the experimental and technical facilities, and valuable discussions of the main results of the present study.

\section{REFERENCES}

[1] A. P. Levanyuk and B. A. Strukov, "Ferroelectric Phenomena in Crystals: Physical Foundations," Berlin: Springer, 1998.

[2] A. P. Levanyuk, V. V. Osipov, A. S. Sigov, and A. A. Sobyanin, "Change of defect structure and the resultant anomalies in the properties of substances near phase-transition points," Sov. Phys. JETP, vol. 49, pp. 176-188, 1979.

[3] E. Sandvold and E. Courtens, "Logarithmic correction of the electric susceptibility in paraelectric tris-sarcosine calcium chloride," Phys. Rev. B, vol. 27, pp. 5660-5668, 1983.

[4] F. D. Morrison, D. C. Sinclair, and A. R. West, "Electrical and structural characteristics of lanthanum-doped barium titanate ceramics," J. Appl. Phys., vol. 86, pp. 6355-6366, 1999.

[5] S. M. Pilgrim, A. E. Sutherland, and S. R. Winzer, "Diffuseness as a useful parameter for relaxor ceramics," J. Amer. Ceram. Soc., vol. 73, pp. 3122-3125, 1990.

[6] R. Shopa, I. Girnyk, Y. Klymovych, O. Kushnir, and V. Tsyumra, "Nonlinear fitting of the critical behavior of dielectric permittivity and heat capacity in ferroelectric crystals with phase transitions of the second order," Scientific Herald of Uzhhorod University. Series Physics, vol. 34, pp. 13-21, 2013.

[7] I. S. Girnyk, Y. G. Klymovych, O. S. Kushnir, and R. Y. Shopa, "Dielectric permittivity of nonstoichiometric lead germanate crystals," Ferroelectrics, vol. 462, pp. 55-63, 2014.

[8] O. S. Yermakov and V. M. Duda, "Nonstoichiometry and conductivity anisotropy of lead germanate single crystals," Ukr. J. Phys., vol. 55, pp. 218-221, 2010.

[9] Y. G. Klymovych, O. S. Ermakov, and O. S. Kushnir, "Nonlinear approximation of temperature dependence of dielectric permittivity of non-stoichiometric ferroelectrics $\mathrm{Pb}_{4.95} \mathrm{Ge}_{3} \mathrm{O}_{11}$," Materials of VIII Ukrainian-Polish Conference "Electronics and Information Technologies" (Lviv-Chynadiyevo, Ukraine, August 27-30, 2016). Lviv: Publishing House of Ivan Franko National University of Lviv, pp. 199-203, 2016. 